Virachit Khy Ṃ, Michel Girard MD MHPE FRCPC

\section{L'utilisation de la 2-chloroprocaïne pour les blocs combinés du plexus lombaire et du nerf sciatique}

ration with this combination of blocks. Twenty-five patients ASA $I$ and 2 were studied. The techniques used have been described by Winnie and Labat. A total dose of $60 \mathrm{ml}$ of 2CP 3\% was injected. Latency time before anaesthesia was approximately seven min for the lumbar plexus and $14 \mathrm{~min}$ for the sciatic nerve. The mean duration of the motor block was $71 \pm 16 \mathrm{~min}, 76.8 \pm 15.3 \mathrm{~min}$ and $75 \pm 12.7 \mathrm{~min}$ for the sciatic, femoral and obturator nerves respectively. The mean duration of the sensory block was $88.7 \pm 20.9 \mathrm{~min}, 83.3 \pm$ 16.4, 79.7 $\pm 17.8 \mathrm{~min}$ and $93.7 \pm 22 \mathrm{~min}$ for the sciatic, femoral, obturator and lateral femoral cutaneous nerves respectively. Success rate was $92 \%$ and no major complication occurred. We conclude that a combination of the lumbar plexus and the sciatic nerve blocks with 2-CP is a useful technique for ambulatory surgery of short duration.

Depuis le début du siècle, les techniques d'anesthésie loco-régionale utilisées pour la chirurgie du membre inférieur sont la rachidienne et l'épidurale. Ces techniques dites centrales comportent des désavantages comme les perturbations hémodynamiques, la céphalée postponction de la dure-mère et la rétention urinaire. Certains de ces inconvénients font hésiter plusieurs anesthésistes à utiliser ces techniques en chirurgie ambulatoire. Une technique anesthésique régionale de courte durée serait donc un ajout intéressant à notre pratique.

Pour l'anesthésie destinée à des interventions de chirurgie ambulatoire, nous sommes à la recherche d'un anesthésique local à début d'action rapide, de courte durée (surtout pour le bloc moteur) et à faible toxicité afin de permettre l'administration du grand volume nécessaire à la réussite du bloc. Parmi les études ${ }^{1,2}$ publiées sur l'utilisation de différents anesthésiques locaux pour le bloc lombo-sciatique, aucune n'a porté sur l'utilisation exclusive de la 2-chloroprocaine (2-CP).

Cette étude a pour objet de documenter l'usage de la 2-CP dans le bloc combiné du plexus lombaire et du nerf sciatique lors dinterventions de courte durée du membre inférieur en chirurgie ambulatoire. 


\section{Méthodologie}

Le protocole a reçu l'approbation des comités de recherche et d'ethique du centre hospitalier. Une autorisation éclairée et écrite a été obtenue de chaque patient. Vingt-cinq patients, ASA I et II, admis en chirurgie ambulatoire pour une intervention d'une durée de moins de 60 min à un membre inférieur ont participé à l'étude (Tableau I). Les critères d'exclusion étaient la présence d'une coagulopathie, d'une neuropathie progressive, d'allergie au PABA, de pseudocholinestérases atypiques ou d'une atteinte infectieuse systémique.

A l'arrivée en salle d'opération, les moniteurs (ECG, $\mathrm{SpoO}_{2}$ et NIPA) et un soluté sont installés et de l'oxygène administré. Par la suite le patient est placé en décubitus latéral. Du midazolam $\left(\leq 0,05 \mathrm{mg} \cdot \mathrm{kg}^{-1}\right)$ et du fentanyl (1-2 $\left.\mu \mathrm{g} \cdot \mathrm{kg}^{-1}\right)$ sont administrés pour le confort du patient. Sous la supervision d'un anesthésiste expérimenté, un résident junior réalise les blocs. Les points de repère sont tracés au crayon dermographique. Après la désinfection et l'infiltration cutanée, le nerf sciatique est repéré par neurostimulation suivant l'approche de Labat ${ }^{3}$ modifiée par Winnie. ${ }^{4}$ Les mouvements du pied et des orteils sont recherchés à 1,0 mA. Lorsque l'amplitude maximale de ce mouvement, à une intensité égale ou inférieure à $0,5 \mathrm{~mA}$, est atteinte, la 2-CP 3\% est injectée en doses fractionnées avec test d'aspiration à tous les $5 \mathrm{ml}$. La position du patient, de même que les points de repère pour effectuer les deux blocs sont illustrés sur la Figure.

Le bloc du plexus lombaire est réalisé selon l'approche postérieure décrite par Winnie ${ }^{4}$ à l'aide d'un neurostimulateur. $^{5} \mathrm{La}$ contraction du quadriceps est recherchée par neurostimulation tel que décrit plus haut.

Une dose totale de $60 \mathrm{ml}$ de 2-CP $3 \%$ est utilisée, et la répartition des volumes pour chaque bloc dépend du site de la chirurgie. Pour la chirurgie du genou, 35 $\mathrm{ml}$ sont utilisés pour le bloc du plexus lombaire et 25 pour le nerf sciatique. Pour la chirurgie distale au genou les volumes utilisés sont intervertis. Les données suivantes sont conservées: le temps pour effectuer les bloc sciatique et lombaire, le temps requis pour l'apparition des blocs moteur et sensitif des nerfs sciatique, fémoral, obturateur et fémoro-cutané (sensitif), le site de la chirurgie et sa durée, la durée des blocs moteur et sensitif des nerfs sciatique, fémoral, obturateur et fémoro-cutané (sensitif). Une échelle ${ }^{6}(0=$ aucune contraction musculaire, $1=$ mouvements à peine perceptibles, $2=$ mouvements actifs en enlevant la pesanteur, $3=$ mouvements contre la gravité, $4=$ mouvements contre la gravité et une certaine résistance, $5=$ force musculaire normale) pour évaluer le bloc moteur est utilisée, un bloc moteur est présent si l'evaluation du bloc moteur est égale ou inférieure à 1 . La perte de sensibilité au froid sert à évaluer l'apparition
TABLEAU I Principales données démographiques (moyenne \pm écart type)

\begin{tabular}{lc}
\hline Age (ans) & $38,8 \pm 18,3$ \\
Fermmes $(n)$ & 15 \\
Hommes $(n)$ & 10 \\
Taille $(\mathrm{cm})$ & $171,0 \pm 9,5$ \\
Poids $(\mathrm{kg})$ & $76,2 \pm 16,0$ \\
\hline
\end{tabular}

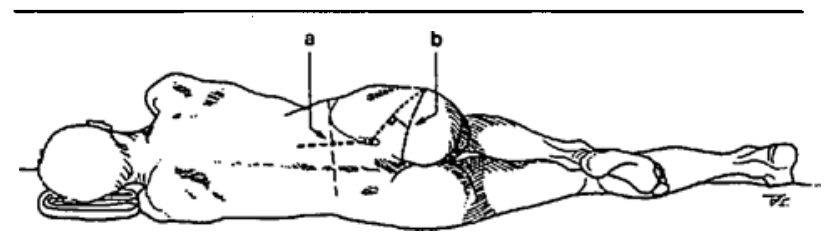

FIGURE Position du patient et points de repère pour l'exécution des blocs du plexus lombaire (a) et du nerf sciatique (b).

TABLEAU II Délai d'apparition du bloc moteur et sensitif en minutes (moyenne \pm écart type)

Nerf sciatique Nerf fémoral Nerf obturateur Nerf fémoro-cutané

Bloc moteur

$13,6 \pm 4,1 \quad 6,9 \pm 3,1 \quad 7,6 \pm 3,8$

Bloc sensitif

$13,9 \pm 6,3 \quad 7,2 \pm 3,2 \quad 7,2 \pm 3,7 \quad 8,3 \pm 5,0$

du bloc sensitif. On note l'utilisation d'un garrot et la présence ou absence d'inconfort reliée à son utilisation.

À la fin de la chirurgie, nous avons expliqué et remis aux patients une échelle visuelle analogique de douleur de $10 \mathrm{~cm}(0=$ aucune douleur, $10 \mathrm{~cm}=$ douleur insupportable) afin d'évaluer leur douleur au moment de l'appel téléphonique du lendemain. Comme convenu, nous avons communiqué avec les patients 24 heures après l'intervention afin d'évaluer la fréquence des lombalgies et leur gravité.

Un test de $\mathrm{t}$ de Student a été utilisé et une valeur de $P<0.05$ est jugée significative.

\section{Résultats}

Les données démographiques des patients se trouvent au tableau I. La sédation se compose en moyenne de midazolam $0,025 \mathrm{mg} \cdot \mathrm{kg}^{-1}$ et de fentanyl $1,0 \mu \mathrm{g} \cdot \mathrm{kg}^{-1}$. Aucun analgésique supplémentaire n'a été nécessaire chez 22 des 25 patients $(92 \%)$.

Le temps moyen requis pour effectuer le bloc sciatique est de 4,24 $\pm 1,81 \mathrm{~min}$ et de 5,86 $\pm 2,59$ minutes pour le plexus lombaire. Le temps moyen (Tableau II) requis pour l'apparition du bloc moteur est de 13,65 $\pm 4,11$ min pour le nerf sciatique, de $6,92 \pm 3,19$ pour le fémoral 
et de 7,60 $\pm 3,85 \mathrm{~min}$ pour l'obturateur. En moyenne, le bloc sensitif est apparu en 13,93 $\pm 6,33$ min pour le sciatique, et 7,22 $\pm 3,20 \mathrm{~min}$ pour le fémoral, en 7,22 $\pm 3,76 \mathrm{~min}$ pour l'obturateur et en $8,33 \pm 5,0 \mathrm{~min}$ pour le fémoro-cutané.

Quinze des vingt-cinq patients ont subi des arthroscopies, les dix autres ont eu des chirurgies de la jambe ou du pied. La durée moyenne de la chirurgie est de $33,1 \pm 14,07 \mathrm{~min}$.

La durée moyenne (Tableau III) du bloc moteur du sciatique est de $71,00 \pm 16,08 \mathrm{~min}$, de 76,85 $\pm 15,36$ min pour le fémoral, de $75,08 \pm 12,70 \mathrm{~min}$ pour l'obturateur. La durée moyenne des blocs sensitifs est supérieure à celle des blocs moteurs. Elle est de 88,74 \pm $20,95 \mathrm{~min}$ pour le sciatique $(P<0.001)$, de $83,33 \pm$ 16,47 pour le fémoral $(P<0.05)$, de $79,74 \pm 17,82$ pour l'obturateur $(P<0.05)$ et finalement de 93,76 $\pm 22,06$ pour le fémoro-cutané. On a utilisé un garrot chez 21 des 22 patients. La durée d'utilisation est comparable à celle de la chirurgie. Aucun patient n'a noté d'inconfort dû au garrot.

Le lendemain de lintervention, trois des 22 patients se sont plaints de malaises ou d'inconfort au site de ponction mais aucune lombalgie évaluée à plus de trois sur dix sur l'échelle visuelle analogique n'a été notée. Ces malaises ont disparu en moins de 24 heures. Nous avons perdu le suivi de trois de nos patients.

\section{Discussion}

L'anesthésie loco-régionale périphérique unilatérale était rarement utilisée pour la chirurgie du membre inférieur avant la description du bloc du plexus lombaire par Winnie. ${ }^{7}$ Cette impopularité de l'anesthésie régionale unilatérale du membre inférieur venait du fait qu'il fallait exécuter plusieurs blocs, soi ceux des nerfs fémoral, obturateur, fémoro-cutané et sciatique, et qu'un de ceuxci, le bloc de l'obturateur, était particulièrement difficile à réaliser. Le but des différentes techniques plus récentes de bloc plexique du membre inférieur est de produire une anesthésie unilatérale à l'aide d'un nombre réduit de ponctions tout en évitant les plus difficiles.

Le bloc du plexus lombaire répond à ces critères, car il permet d'anesthésier trois des quatre principaux nerfs de la jambe avec une seule injection. ${ }^{4}$ La recherche de paresthésies est utilisée pour l'approche postérieure du plexus lombaire appelée par Winnie " bloc du plexus lombo-sacré ". Selon cet auteur, cette technique permettrait le bloc concomitant des racines sacrées. Chayen, ${ }^{8}$ deux ans plus tard, décrit le bloc du plexus lombaire, grâce à des repères anatomiques différents avec une technique utilisant la perte de résistance qu'il surnomme le " psoas compartment block ". Par la suite, Dalens ${ }^{5}$ publie la première étude comparative des deux approches pos-
TABLEAU III Durée du bloc moteur et sensitif en minutes (moyenne \pm écart type)

\begin{tabular}{llll}
\hline Nerf sciatique* $^{*}$ & Nerf fëmoral $\dagger$ & Nerf obturateur $\dagger$ Nerf fémoro-cutané \\
\hline $\begin{array}{l}\text { Bloc moteur } \\
71 \pm 16\end{array}$ & $76,8 \pm 15,3$ & $75 \pm 12,7$ \\
Bloc sensitif & & & \\
$88,7 \pm 20,9$ & $83,3 \pm 16,4$ & $79,7 \pm 17,8$ & $93,7 \pm 22$ \\
\hline
\end{tabular}

${ }^{*} P<0.001 . \dagger P<0.05$

térieures du plexus lombaire, soit le bloc du plexus lombosacré de Winnie et le bloc du compartiment du psoas de Chayen en utilisant un neurostimulateur pour les deux techniques. Il conclut que le taux de succès est comparable et acceptable pour les deux approches. Cependant, l'approche de Chayen, modifiée par Dalens, est plus difficile à réaliser et s'accompagne un pourcentage élevé de blocs bilatéraux.

Parkinson, ${ }^{9}$ en 1989, compare l'approche postérieure du plexus lombaire et l'approche antérieure communément appelée " bloc 3-en-1 ${ }^{7}$ En se basant sur l'hypothèse que l'innervation sensitive du nerf obturateur est très variable et que seul un bloc moteur de ce dernier prouve l'efficacité de la technique, il conclut que l'approche postérieure est supérieure à l'approche antérieure pour le nombre de nerfs bloqués. Cependant, aucune technique de bloc du plexus lombaire n'ayant permis de bloquer de façon fiable les territoires innervés par le sciatique, Parkinson suggère le bloc combiné du plexus lombaire et du nerf sciatique pour l'anesthésie unilatérale du membre inférieur.

Nous avons choisi d'utiliser l'approche postérieure du bloc du plexus lombaire, car elle est de technique facile et peut être réalisée alors que le patient est placé dans la même position que pour le bloc du nerf sciatique. De plus l'approche postérieure se révèle à tout le moins aussi efficace que l'approche antérieure. ${ }^{9}$

Nous avons commencé par le bloc du nerf sciatique parce que notre expérience clinique nous indiquait que le temps nécessaire pour l'installation du bloc du nerf sciatique était plus long. Effectivement, cette étude corrobore notre impression clinique.

Le début d'action de la 2-CP semble être plus rapide que celui des autres anesthésiques locaux rapportés dans la littérature puisque son bloc moteur s'est installé en $8,9 \mathrm{~min}$ comparativement à $14,2 \mathrm{~min}$ pour la mépivacaïne $^{10}$ et 16,4 min pour la bupivacaïne. ${ }^{10}$ Etant donné que sa durée d'action (surtout pour le bloc moteur) est apparue relativement courte ( $71 \mathrm{~min}$ pour le nerf sciatique et 76 pour le nerf fémoral), la 2-CP est un anesthésique local intéressant pour les patients de chirurgie ambula- 
toire chez qui un retour rapide de la motricité est essentiel.

Le pourcentage de succès ( $92 \%$ ) obtenu par cette technique dans notre étude est semblable à ceux des études antérieures. ${ }^{5,9}$ Seulement trois patients sur 25 ont eu besoin d'une anesthésie supplémentaire. Pour le patient $\mathrm{n}^{\circ}$ 9, on a constaté un bloc moteur et sensitif incomplet du nerf obturateur après 20 min malgré une neurostimulation adéquate du quadriceps. Un supplément de 100 $\mu \mathrm{g}$ de fentanyl et une perfusion de propofol à 25 $\mu \mathrm{g} \cdot \mathrm{kg}^{-1} \cdot \mathrm{min}^{-1}$ ont été administrés. Le patient $\mathrm{n}^{\circ} 24$ a présenté un bloc moteur et sensitif incomplet dans les territoires du nerf fémoral et obturateur malgré la neurostimulation évidente du quadriceps. Une absence d'anesthésie a également été constatée dans le territoire du fémoro-cutané. Chez ce patient la réalisation du bloc a été difficile et la stimulation à $0,5 \mathrm{~mA}$ était très faible. Une anesthésie générale a été nécessaire pour la chirurgie arthroscopique. Le patient $n^{\circ} 26$ était obèse et la technique fut laborieuse. De plus, l'aiguille s'est déplacée après le début du linfiltration du sciatique de sorte que ce nerf était incomplètement bloqué après $20 \mathrm{~min}$. Nous avons procédé à une anesthésie générale.

Quatre raisons ont motivé notre décision de n'utiliser qu'une seule dose de 2-CP pour tous nos patients. Premièrement, nombre d'études utilisant des doses d'anesthésiques locaux, supérieures à celles recommandées, ont été publiées sans rapporter de complications sérieuses. ${ }^{1,11-14}$ Deuxièmement, la dose totale de $1800 \mathrm{mg}$ que nous utilisons sans tenir compte du poids du patient, peut dans certains cas dépasser la dose maximale recommandée par le fabricant $\left(11 \mathrm{mg} \cdot \mathrm{kg}^{-1}\right)$ et par la plupart des manuels d'anesthésie. ${ }^{15,16} \mathrm{La}$ dose maximale recommandée par Covino, ${ }^{17}$ l'auteur le plus souvent cité sur ce sujet, est de $22,8 \mathrm{mg} \cdot \mathrm{kg}^{-1}$. Cette recommandation est basée sur une étude de Foldes ${ }^{18}$ publiée en 1960. Dans cette étude, où l'on a perfusé de la 2-CP à raison de $1 \mathrm{mg} \cdot \mathrm{kg}^{-1} \min \dot{N}, 10$ patients sur 12 ont terminé l'expérience dune durée prédéterminée de $25 \mathrm{~min}$. La perfusion était cessée à l'apparition de fasciculations généralisées, de convulsions, de difficultés respiratoires graves, de pertes de conscience ou d'une désorientation complète. La moyenne de la durée de perfusion étant de 22,8 \pm $1,3 \mathrm{~min}$, on en a extrapolé la dose maximale recommandée de $22,8 \mathrm{mg} \cdot \mathrm{kg}^{-1}$. Dans l'étude de Foldes les symptômes d'absorption sont apparus à 3,0 $\pm 0,4 \mathrm{~min}$ après le début et disparus à $4,74 \pm 1,3$ min après l'arrêt de la perfusion. Parmi les 12 patients observés, sept ont présenté des fasciculations ou des contractions musculaires mais aucun n'a présenté de convulsions. Dans la majorité des cas, la T.A. et le pouls sont demeurés inchangés ou ont augmenté. Nous n'avons pas mesuré les concentrations sériques de 2-CP. Par ailleurs, aucune dépression respiratoire n'a été constatée dans l'étude de Foldes et l'ECG n'a pas changé dans la majorité des cas. Des travaux chez le chien ${ }^{19}$ ont montré qu'un bolus rapide et unique de $30 \mathrm{mg} \cdot \mathrm{kg}^{-1}$ de 2-CP pouvait être létal et que la dose létale cumulative était de $57 \mathrm{mg} \cdot \mathrm{kg}^{-1}$. Or, Aven et al. rapportaient en 1953 (Federation Proceedings abstract 986) que l'hydrolyse des esters est plus lente chez le chien que chez l'homme. Même si l'application de ces données à l'humain doit être faite avec prudence, il reste néanmoins que la dose maximale recommandée ne repose sur aucune donnée bien documentée.

Troisièmement, différentes publications ont montré que les concentrations sériques mesurées ne sont pas corrélées au poids du patient pour une dose fixe d'anesthésique local donné. ${ }^{20-23}$

Quatrièmement, l'absorption intravasculaire d'anesthésique local pendant le bloc lombo-sciatique est vraisemblablement similaire à celle qui se produit lors d'une technique de bloc combiné du nerf fémoral et du nerf sciatique. Or Moore ${ }^{12}$ a publié qu'après les blocs du nerf fémoral et du nerf sciatique les taux sériques mesurés étaient semblables à ceux obtenus lors de blocs du plexus brachial. C'est en tenant compte de tous ces facteurs que nous avons décidé d'utiliser une dose identique pour tous les patients.

L'utilisation de 2-CP $2 \%$ aurait pu permettre de diminuer la dose d'anesthésique local. Cependant notre expérience nous a montré que, pour ces blocs, cette concentration ne produisait pas une anesthésie satisfaisante.

Nous savons que des lombalgies apparaissant moins de 24 heures après l'épidurale à la $2-\mathrm{CP}$, ont été rapportés. ${ }^{24-27}$ L'agent responsable de ces spasmes musculaires intenses, et difficilement contrôlés, serait l'EDTA (ethylene diamine tetraacetic acid), ${ }^{29}$ utilisé comme agent de conservation. Nous avons communiqué avec les patients par téléphone 24 heures après l'intervention. Aucun patient n'a présenté de lombalgies dépassant 3/10 sur l'échelle visuelle analogique. Quatre des 22 patients ont rapporté des malaises au site dinjection qui sont disparus en moins de 24 heures, avec une intensité variant de 2 à 3. Aucun des patients ne s'est plaint de sciatalgie et aucun déficit neurologique n'a été noté au moment du départ. Il ne semble pas que la 2-CP utilisée ait un effet toxique sur les muscles comme Fibush et Opper ${ }^{25}$ l'ont proposé puisquil n'y a pas eu de lombalgies graves bien que linjection se fasse à proximité des muscles.

Un patient a développé un bloc bilatéral dans le territoire du plexus lombaire. On notait du côté opéré un bloc moteur et sensitif complet de trois principaux nerfs du plexus lombaire et du sciatique. Un bloc moteur et sensitif complet du plexus lombaire épargnant le territoire du sciatique, est apparu du côté opposé. Le niveau sensitif maximal atteint était de $T_{10}$ du côté opéré et de $T_{12}$ de 
l'autre. Vingt-cinq $\mathrm{ml}$ de 2-CP $3 \%$ avaient été utilisés pour bloquer le plexus lombaire. Lincidence de bloc bilatéral, dans ce travail, est similaire à celle retrouvée dans les séries antérieures. ${ }^{9,28}$ Par contre, le mécanisme du bloc bilatéral n'est pas encore définitivement élucidé. Dalens ${ }^{5}$ a proposé que l'anesthésique local pourrait diffuser dans l'espace épidural par extension de cet espace au-delà des trous de conjugaison. La ponction d'un manchon duremérien pourrait également expliquer ce phénomène. On sait que lors de blocs intercostaux ou paravertébraux, un bloc bilatéral est possible. Le mécanisme de ce phénomène est incertain mais il a été démontré que les espaces paravertébraux pouvaient communiquer entre eux par un espace prévertébral. L'anesthésique local ne pénètrerait donc pas nécessairement dans l'espace péridural. ${ }^{30,31}$ Il est important de surveiller le bloc bilatéral et d'utiliser des solutions homologuées pour usage épidural.

Un des patients a présenté des symptômes d'absorption. Vers la fin de l'injection du plexus lombaire, il s'est plaint d'étourdissements évoluant vers la somnolence. Il a présenté des fasciculations au niveau des membres. Cependant, il a toujours maintenu le contact avec nous. Sa respiration est restée normale. Pour ce patient, la dose de $1800 \mathrm{mg}$ de 2 -CP équivalait à $20 \mathrm{mg} \cdot \mathrm{kg}^{-1}$. La pression artérielle et le pouls ont augmenté. Ce phénomène d'absorption est survenu malgré des tests d'aspiration répétés et négatifs. Tous les symptômes sont disparus en 2-3 min. L'intervention s'est déroulée sans problème et sans séquelle. Nous avons remarqué que la résistance à l'injection avait été plus grande qu'habituellement. La technique complète, comprenant le repérage du plexus et l'injection des $35 \mathrm{ml}$ de 2-CP $3 \%$, a duré moins de $3 \mathrm{~min}$. Il est à noter que dans l'étude de Foldes ${ }^{18}$ les symptômes d'absorption sont apparus 3 min après la perfusion et le délai d'apparition des symptômes ne nous renseigne pas sur la quantité absorbée. Afin de limiter les risques de ce genre de complication deux mesures pourraient être utiles: une réduction de la vitesse d'injection et l'ajout d'épinéphrine à l'anesthésique local.

En conclusion, lutilisation de la 2-CP lors du bloc du plexus lombaire et du nerf sciatique pour la chirurgie du membre inférieur s'est avérée efficace et pratique. Peu d'effets secondaires importants ont été notés. Cependant, afin d'administrer la dose minimale efficace et d'en réduire l'absorption, l'anesthésiste devrait considérer l'ajout d'adrénaline à la 2-CP.

\section{Remerciements}

Les auteurs souhaitent remercier Pierre Drolet MD et Edith Villeneuve MD pour leur précieuse assistance de même que Juliana Farny MD pour la figure.

\section{Références}

1 Simon $M A$, Gielen $M J$, Lagerwerf $A J$, Vree TB. Plasma concentrations after high doses of mepivacaine with epinephrine in the combined psoas compartment/sciatic nerve block. Reg Anesth 1990; 15: 256-60.

2 Vaghadia H, Kapnoudhis P, Jenkins LC, Taylor D. Continuous lumbosacral block using a Tuohy needle and catheter technique. Can J Anaesth 1992; 39: 75-8.

3 Labat G. Regional Anesthesia - Its technic and clinical application. Philadelphia: W.B. Saunders, 1923.

4 Winnie AP, Ramamurthy $S$, Durrani $Z$, Radonjic $R$. Plexus blocks for lower extremity surgery. Anesthesiology Review 1974; 11-6.

5 Dalens B, Tanguy A, Vanneuville $G$. Lumbar plexus block in children: a comparison of two procedures in 50 patients. Anesth Analg 1988; 67: 750-8.

6 Bates $B$. Guide de l'examen clinique, $2^{\text {me }}$ ed. Paris: Medsi, 1985.

7 Winnie AP, Ramamurthy S, Durrani $Z$. The inguinal paravascular technic of lumbar plexus anesthesia: the " 3 -in-1 block." Anesth Analg 1973; 52: 989-96.

8 Chayen D, Nathan H, Chayen $M$. The psoas compartment block. Anesthesiology 1976; 45: 95-9.

9 Parkinson SK, Mueller JB, Little WL, Bailey SL. Extent of blockade with various approaches to the lumbar plexus. Anesth Analg 1989; 68: 243-8.

10 Moore DC, Bridenbaugh LD, Bridenbaugh PO, Tucker GT. Bupivacaine for peripheral nerve block: a comparison with mepivacaine, lidocaine, and tetracaine. Anesthesiology 1970; 32: 460-3.

11 Moore DC, Mather LE, Bridenbaugh LD, Balfour RI, Lysons $D F$, Horton WG. Arterial and venous plasma levels of bupivacaine following peripheral nerve blocks. Anesth Analg 1976; 55: 763-8.

12 Moore DC, Bridenbaugh LD, Thomspon GE, Balfour RI, Horton WG. Bupivacaine: a review of 11,080 cases. Anesth Analg 1978; 57: 42-53.

13 Neill RS, Watson R. Plasma bupivacaine concentrations during combined regional and general anaesthesia for resection and reconstruction of head and neck carcinomata. $\mathrm{Br}$ J Anaesth 1984; 56: 485-92.

14 Tuominen $M$, Rosenberg PH, Kalso PH. Blood levels of bupivacaine after single dose, supplementary dose and during continuous infusion in axillary plexus block. Acta Anaesthesiol Scand 1983; 27: 303-6.

15 Carpenter RL, Mackey DC. Local anesthetics. In: Barash PG, Cullen BF, Stoelting RK (Eds.). Clinical Anesthesia, 2nd ed. Philadelphia: J.B. Lippincott Co., 1992; 509-41.

16 Denson DD. Physiology, pharmacology and toxicity of local anesthetics: adult and pediatric considerations. In: Raj PP (Ed.). Clinical Practice of Regional Anesthesia. New York: Churchill Livingstone Inc, 1991; 73-105.

17 Covino BG, Vassallo HG. Local Anesthetics: Mechanisms 
of Action and Clinical Use. New York: Grune \& Stratton, 1976.

18 Foldes FF, Molloy R, McNall PG, Koukal LR. Comparison of toxicity of intravenously given local anesthetic agents in man. JAMA 1960; 172: 1493-8.

19 Liu P, Feldman HS, Covino BM, Giasi R, Covino BG. Acute cardiovascular toxicity of procaine, chloroprocaine, and tetracaine in anesthetized ventilated dogs. Reg Anesth 1982; 7: 14-9.

20 Rowland $M$, Thomson PD, Guichard A, Melmon $K L$. Disposition kinetics of lidocaine in normal subjects. Ann $\mathrm{N}$ Y Acad Sci 1971; 179: 383-98.

21 Tucker GT, Mather LE. Pharmacokinetics of local anaesthetic agents. Br J Anaesth 1975; 47: 213-24.

22 Scott DB, Cousins MJ. Clinical pharmacology of lcoal anesthetic agents. In: Cousins MJ, Bridenbaugh PO (Eds.). Neural Blockade. Philadelphia: J.B. Lippincott Co., 1980; 86-121.

23 Pihlajamäki KK. Inverse correlation between the peak and venous serum concentration of bupivacaine and the weight of the patient during interscalene brachial plexus block. $\mathrm{Br}$ J Anaesth 1991; 67: 621-2.

24 Levy L, Randel GI, Pandit SK. Does chloroprocaine (Nesacaine MPF) for epidural anesthesia increase the incidence of backache? (Letter). Anesthesiology 1989; 71: 476.

25 Fibuch EE, Opper SE. Back pain following epidurally administered Nesacaine-MPF. Anesth Analg 1989; 69: 113-5.

26 Hynson JM, Sessler DI, Glosten B. Back pain in volunteers after epidural anesthesia with chloroprocaine. Anesth Analg 1991; 72: 253-6.

27 McLoughlin TM, DiFazio CA. More on back pain after Nesacaine-MPF (Letter). Anesth Analg 1990; 71: 562-3.

28 Dalens $B$, Vanneuville $G$. Tanguy $A$. Comparison of the fascia iliaca compartment block with the 3-in-1 block in children. Anesth Analg 1989; 69: 705-13.

29 Mowbray A, Wong KKS, Murray JM. Intercostal catheterization. An alternative approach to the paravertebral space. Anaesthesia 1987; 42: 958-61.

30 Middaugh RE, Menk EJ, Reynolds WJ, Bauman JM, Cawthon MA, Hartshorne MF. Epidural block using large volumes of local anesthetic solution for intercostal nerve block. Anesthesiology 1985; 63: 214-6.

31 Conacher ID, Kokri M. Postoperative paravertebral blocks for thoracic surgery. A radiological appraisal. Br J Anaesth 1987; 59: 155-61. 